\title{
SEMIGROUPS SATISFYING VARIABLE IDENTITIES. II
}

\author{
BY \\ MOHAN S. PUTCHA AND JULIAN WEISSGLASS
}

\begin{abstract}
The concept of a semigroup satisfying an identity $x y=f(x, y)$ is generalized by considering identities in $n$-variables and letting the identity depend on the variables. The property of satisfying a "variable identity" is studied. Semigroups satisfying certain types of identities are characterized in terms of unions and semilattices of groups.
\end{abstract}

Introduction. Semigroups satisfying an identity of the form $x y=f(x, y)$ have been studied by Tully [5] and Tamura [4]. In [2], we generalized Tamura's result. We considered semigroups $S$ satisfying: for every $a, b \in S$ there exists a positive integer $m$ such that $a b=b^{\lambda_{1}} a^{\mu_{1}} \cdots b^{\lambda_{m}} a^{\mu_{m}}$ where $\lambda_{i}, \mu_{i}$ are integers greater than one, $i=1, \ldots, m$, and $\sum_{i=1}^{m} \mu_{i} \geqq 2$. We proved that a semigroup $S$ satisfies this condition if and only if $S$ is an inflation of a semilattice of periodic groups. The purpose of this article is to consider semigroups satisfying the analogous condition for $n$ variables.

1. Preliminaries. Throughout $S$ will denote a semigroup and $E=E(S)$ the set of idempotents of $S$ and $n$ will be an integer, $n \geqq 2$. Let $F_{n}$ denote the free (noncommutative) semigroup generated by the distinct letters $x_{1}, \ldots, x_{n}$. Denote by $C_{n}$ the subsemigroup of $F_{n}$ consisting of all elements $x \in F_{n}$ each of which is the product of all of the $x_{1}, \ldots, x_{n}$, allowing repeated use. Let $R_{n}$ denote the semigroup ring of $C_{n}$ over the integers $Z$. Thus $R_{n}$ is the set of functions of finite support from $C_{n}$ to $Z$. It is well known that $R_{n}$ can be considered as the set of finite formal sums of elements in $C_{n}$ and coefficients in $Z$.

Definition. We define a subset $\mathscr{V}_{n}$ of $R_{n}$ by $\mathscr{V}_{n}=\left\{f \mid f \in R_{n}, f=f_{1}-f_{2}\right.$, there exists $c_{1}, c_{2} \in C_{n}, c_{1} \neq c_{2}$ such that $f_{i}(c)=0$ unless $\left.c=c_{i}, f_{i}\left(c_{i}\right)=1\right\}$.

Thus $\mathscr{V}_{n}$ is the set of all $f \in R_{n}$ which are the differences of two different monomials. That is, $f \in \mathscr{V}_{n}$ if and only if $f=x_{k_{1}} \cdots x_{k_{r}}-x_{l_{1}} \cdots x_{l_{s}} \neq 0$, where each $x_{i}$ $(i=1, \ldots, n)$ appears at least once in $x_{k_{1}} \cdots x_{k_{r}}$ and at least once in $x_{l_{1}} \cdots x_{l_{s}}$.

If $a_{1}, \ldots, a_{n} \in S$ there exists a homomorphism $\varphi: C_{n} \rightarrow S$ such that $\varphi\left(x_{i}\right)=a_{i}$. This homomorphism extends to a homomorphism $\varphi^{\prime}$ from $R_{n}$ into the semigroup ring of $S$ over $Z$. If $f=f_{1}-f_{2} \in \mathscr{V}_{n}$ and $a_{1}, \ldots, a_{n} \in S$ we say that $f_{1}\left(a_{1}, \ldots, a_{n}\right)$ $=f_{2}\left(a_{1}, \ldots, a_{n}\right)$ if $f \in \operatorname{ker} \varphi^{\prime}$. Thus $f_{1}\left(a_{1}, \ldots, a_{n}\right)=f_{2}\left(a_{1}, \ldots, a_{n}\right)$ if and only if considering $f_{1}$ and $f_{2}$ as monomials in $x_{1}, \ldots, x_{n}$ when $a_{i}$ is substituted for $x_{i}$ and

Received by the editors April 19, 1971.

AMS 1969 subject classifications. Primary 2093.

Key words and phrases. Semigroups, variable identity, semilattice, inflation.

Copyright (C) 1972, American Mathematical Society 
multiplication performed in $S$, we have $f_{1}\left(a_{1}, \ldots, a_{n}\right)=f_{2}\left(a_{1}, \ldots, a_{n}\right)$. We will use without further comment this characterization of $f_{1}\left(a_{1}, \ldots, a_{n}\right)=f_{2}\left(a_{1}, \ldots, a_{n}\right)$.

If $\mathscr{X}$ is a subset of $\mathscr{V}_{n}$, we say that $S$ is an $\mathscr{X}$-semigroup if for every $a_{1}, \ldots, a_{n} \in S$ there exists $f=f_{1}-f_{2} \in \mathscr{X}$ (depending on $a_{1}, \ldots, a_{n}$ ) such that

$$
f_{1}\left(a_{1}, \ldots, a_{n}\right)=f_{2}\left(a_{1}, \ldots, a_{n}\right) \text {. }
$$

We prove a preliminary result for arbitrary subsets $\mathscr{X} \subseteq \mathscr{V}_{n}$.

Theorem 1.1. Suppose $T$ is a semilattice $\Omega$ of semigroups $T_{\alpha}, \alpha \in \Omega$, such that each $T_{\alpha}$ has an identity element. Then $T$ is an $\mathscr{X}$-semigroup if and only if each $T_{\alpha}$ is an $\mathscr{X}$-semigroup.

Proof. If $T$ is an $\mathscr{X}$-semigroup then each $T_{\alpha}$ is an $\mathscr{X}$-semigroup since subsemigroups of $\mathscr{X}$-semigroups are $\mathscr{X}$-semigroups. To prove the converse let $a_{1}, \ldots, a_{n} \in T$. Suppose $a_{i} \in T_{\alpha_{i}}$ and let $\alpha=\alpha_{1} \cdots \alpha_{n}$ (the product in the semilattice $\Omega$ ). Then $a=a_{1} \cdots a_{n} \in T_{\alpha}$. Let $e$ be the identity of $T_{\alpha}$. Since $\alpha_{i} \alpha=\alpha \alpha_{i}=\alpha$ we have that $a_{i} e$ and $e a_{i}$ are in $T_{\alpha}$, for $i=1, \ldots, n$. Furthermore $e a_{i}=\left(e a_{i}\right) e=e\left(a_{i} e\right)=a_{i} e$. Now applying the hypothesis to $T_{\alpha}$, there exists $f=f_{1}-f_{2} \in \mathscr{X}$ such that $f_{1}\left(a_{1} e, \ldots, a_{n} e\right)$ $=f_{2}\left(a_{1} e, \ldots, a_{n} e\right)$. Since each $x_{i}$ appears at least once in $f_{1}$ and $f_{2}$ and since $\alpha_{i} \alpha=$ $\alpha \alpha_{i}=\alpha$, it follows that $f_{1}\left(a_{1}, \ldots, a_{n}\right)$ and $f_{2}\left(a_{1}, \ldots, a_{n}\right)$ are in $T_{\alpha}$. Hence $f_{1}\left(a_{1}, \ldots, a_{n}\right)$ $=f_{1}\left(a_{1}, \ldots, a_{n}\right) e=f_{1}\left(a_{1} e, \ldots, a_{n} e\right)=f_{2}\left(a_{1} e, \ldots, a_{n} e\right)=f_{2}\left(a_{1}, \ldots, a_{n}\right) e=f_{2}\left(a_{1}, \ldots, a_{n}\right)$. Therefore $T$ is an $\mathscr{X}$-semigroup.

Definition. Let $S$ be a semigroup, $T$ a subsemigroup of $S$. Then $S$ is an $n$th inflation of $T$ if there exists a homomorphism $\theta: S \rightarrow T$ such that $\theta$ is the identity map on $T$ and for each $a_{1}, \ldots, a_{n} \in S,\left(a_{1} \theta\right) \cdots\left(a_{n} \theta\right)=a_{1} \cdots a_{n}$.

REMARK. The 2nd inflation corresponds to the usual concept of inflation (cf. [1, p. 98]). If $S$ is an $n$th inflation of $T$ then $\left(a_{1} \theta\right) \cdots\left(a_{m} \theta\right)=a_{1} \cdots a_{m}$ for all $m \geqq n$.

Corollary 1.2. Let $\mathscr{X} \subseteq \mathscr{V}_{n}$. Suppose $S$ is an nth inflation of $T$, that $T$ is a semilattice of semigroups $T_{\alpha}$, and that each $T_{\alpha}$ is an $\mathscr{X}$-semigroup with identity. Then $S$ is an $\mathscr{X}$-semigroup.

Proof. Let $a_{1}, \ldots, a_{n} \in S$. Then $a_{1} \theta, \ldots, a_{n} \theta \in T$ so by Theorem 1.1 there exists $f=f_{1}-f_{2} \in \mathscr{X}$ such that $f_{1}\left(a_{1} \theta, \ldots, a_{n} \theta\right)=f_{2}\left(a_{1} \theta, \ldots, a_{n} \theta\right)$. By the above remark, for $j=1,2, f_{j}\left(a_{1}, \ldots, a_{n}\right)=f_{j}\left(a_{1} \theta, \ldots, a_{n} \theta\right)$ since each $x_{i}$ appears at least once in $f_{j}$. Thus $f_{1}\left(a_{1}, \ldots, a_{n}\right)=f_{1}\left(a_{1} \theta, \ldots, a_{n} \theta\right)=f_{2}\left(a_{1} \theta, \ldots, a_{n} \theta\right)=f_{2}\left(a_{1}, \ldots, a_{n}\right)$. Hence $S$ is an $\mathscr{X}$-semigroup.

REMARK. The proof of Corollary 1.2 can be modified to prove that if $S$ is an ideal extension of $T$ by an $\mathscr{X}$-semigroup, then $S$ is an $\mathscr{X}$-semigroup.

Definition. Let $n, i, \alpha$ be positive integers $n \geqq 2,1 \leqq i \leqq n$. Certain subsets of $\mathscr{V}_{n}$ are defined by:

(1) $\mathscr{L}_{n}=\left\{f \mid f=f_{1}-f_{2} \in \mathscr{V}_{n} ; f_{1}=x_{1} \cdots x_{n} ; x_{j}\right.$ appears at least twice in $f_{2}$, $j=1, \ldots, n\}$.

(2) $\mathscr{M}_{n}^{i, \alpha}=\left\{f \mid f=f_{1}-f_{2} \in \mathscr{V}_{n}, f_{1}=x_{1} \cdots x_{n}, x_{i}\right.$ appears at least $\alpha$ times in $\left.f_{2}\right\}$. 
(3) If $\mathscr{X} \subseteq \mathscr{V}_{n}$, then $\overline{\mathscr{X}}=\left\{f \mid f=f_{1}-f_{2} \in \mathscr{X}, f_{2}\right.$ starts with $x_{j} ; j \neq 1$ and ends with $x_{k}$, $k \neq n\}$.

(4) $S$ is an $\mathscr{M}_{n}^{i, \infty}$-semigroup if $S$ is an $\mathscr{M}_{n}^{i, \alpha}$-semigroup for all $\alpha \geqq 2$.

Remark. Let $\mathscr{X}, \mathscr{Y} \subseteq \mathscr{V}_{n}$. Then $\mathscr{X} \subseteq \mathscr{Y}$ implies that: (i) every $\mathscr{X}$-semigroup is a $\mathscr{Y}$-semigroup and (ii) $\overline{\mathscr{X}} \subseteq \overline{\mathscr{Y}}$. Also subsemigroups and homomorphic images of $\mathscr{X}$-semigroups are $\mathscr{X}$-semigroups.

We will now prove several lemmas which are needed for the main theorems.

Let $a_{1}, \ldots, a_{n} \in S$ and suppose $x=a_{k_{1}}^{\mu_{1}} \cdots a_{k_{t}}^{\mu_{t}}$. We say that the length of $x$ in the $a_{j}$ 's is $\sum_{i=1}^{t} \mu_{i}$.

Lemma 1.3. (i) Every $\mathscr{L}_{n}$-semigroup is an $\mathscr{M}_{n}^{i, \infty}$-semigroup.

(ii) Every $\overline{\mathscr{M}}_{n}^{i, 2}$-semigroup is an $\mathscr{M}_{n}^{i, \infty}$-semigroup.

Proof. (i) The proof follows by repeated application of the equation

$$
a_{1} \cdots a_{n}=f_{2}\left(a_{1}, \ldots, a_{n}\right) \text {. }
$$

(ii) Since $\overline{\mathscr{M}}_{n}^{i, \alpha} \subseteq \mathscr{M}_{n}^{i, \alpha}$ it suffices to show that for $\alpha \geqq 2$, every $\overline{\mathscr{M}}_{n}^{i, \alpha}$-semigroup is an $\overline{\mathscr{M}}_{n}^{i, \alpha+1}$-semigroup. Let $a_{1}, \ldots, a_{n} \in S$. Then by (2) $a_{1} \cdots a_{n}=f_{2}\left(a_{1}, \ldots, a_{n}\right)$, where each $a_{j}$ appears at least once on the right-hand side and $a_{i}$ appears at least $\alpha$ times. Since we can apply the hypothesis repeatedly, we may assume, without loss of generality, that the length of $f_{2}$ in the $a_{j}$ 's is greater than $2 n^{2}$. There are two possibilities:

(i) $f_{2}\left(a_{1}, \ldots, a_{n}\right)=u a_{i} h_{1} \cdots h_{n}$ with $u, h_{j} \in\left\langle a_{1}, \ldots, a_{n}\right\rangle, a_{i}$ appearing in at least one $h_{j}$ and the length of $h_{j}$ in the $a_{k}$ 's greater than or equal to $n$.

(ii) $f_{2}\left(a_{1}, \ldots, a_{n}\right)=h_{1} \cdots h_{n} a_{i} u, h_{j}, u$ as in (i).

We assume (i), the proof for (ii) being similar. Applying the $\overline{\mathscr{M}}_{n}^{i, \alpha}$ hypothesis to $\left(u a_{i} h_{1}\right) h_{2} \cdots h_{n}$ we have

$$
\begin{aligned}
\left(u_{1} a_{i} h_{1}\right) h_{2} \cdots h_{n} & =f_{3}\left(u a_{i} h_{1}, h_{2}, \ldots, h_{n}\right)=h_{k_{1}} \cdots h_{k_{s}} u a_{i} h_{1} h_{k_{s+1}} \cdots h_{k_{n}} \\
& =g_{1} \cdots g_{i-1} a_{i} g_{i+1} \cdots g_{n},
\end{aligned}
$$

where each $g_{i}$ is a product of the $a_{j}$ 's and $a_{i}$ appears in at least one $g_{j}$. Again applying the $\overline{\mathscr{M}}_{n}^{i, \alpha}$ hypothesis we have $g_{1} \cdots g_{i-1} a_{i} g_{i+1} \cdots g_{n}=f_{4}\left(g_{1}, \ldots, a_{i}, \ldots, g_{n}\right)$. Now since $a_{i}$ appears in some $g_{j}, a_{i}$ appears at least $\alpha+1$ times on the right-hand side. Hence $a_{1} \cdots a_{n}=f_{2}\left(a_{1}, \ldots, a_{n}\right)=u a_{i} h_{1} \cdots h_{n}=f_{4}\left(g_{1}, \ldots, a_{i}, \ldots, g_{n}\right)$ and the proof is complete.

Lemma 1.4. Suppose that $S$ is a semigroup with zero and that $S$ is an $\mathscr{M}_{n,}^{i \infty}-$ semigroup with no nonzero idempotents. Then $S^{n}=\{0\}$.

Proof. Let $s \in S$. Letting $a_{1}=a_{2}=\cdots=a_{n} \in S$, we have from (2) that $s^{n}=s^{k}$, with $k>n$. Hence $s^{m} \in E(S)$ for some $m, n \leqq m \leqq k$. Thus $s^{m}=0$ and hence $s^{n}=s^{k}=0$. Let $l$ be the least positive integer for which $S^{i-1} s^{l} S^{n-i}=\{0\}$. We prove that the assumption $l>1$ leads to a contradiction. Let $t_{1}, \ldots, t_{n} \in S$. Let $\alpha=n+1$ and define

$$
\begin{aligned}
a_{j} & =s^{l-1} t_{j} & & \text { if } 1 \leqq j<i, \\
& =s^{l-1} t_{i} s^{l-1} & & \text { if } j=i, \\
& =t_{j} s^{l-1} & & \text { if } i<j \leqq n .
\end{aligned}
$$


Since $S$ is an $\mathscr{M}_{n}^{i, \alpha}$-semigroup we have $a_{1} \cdots a_{n}=f_{2}\left(a_{1}, \ldots, a_{n}\right)$ with $a_{i}$ appearing at least $\alpha=n+1$ times on the right. It follows that $s^{l}$ appears at least $n$ times on the right. Isolating the $i$ th $s^{l}$ we see that $f_{2}\left(a_{1}, \ldots, a_{n}\right) \in S^{i-1} s^{l} S^{n-i}=\{0\}$. Thus $s^{l-1} t_{1} \cdots s^{l-1} t_{i} s^{l-1} \cdots t_{n} s^{l-1}=f_{2}\left(a_{1}, \ldots, a_{n}\right)=0$. But the $t_{i}$ 's are arbitrary in $S$. Hence $\left(s^{l-1} S\right)^{n+1}=0$. Now apply the $\mathscr{M}_{n}^{i, \alpha}$ condition with $\alpha=2 n+2$ to the elements $a_{j}=t_{j}, j \neq i$ and $a_{i}=s^{l-1}$ where the $t_{j}$ are arbitrary elements of $S$. We have

$$
t_{1} \cdots t_{i-1} s^{l-1} t_{i+1} \cdots t_{n}=f_{2}\left(t_{1}, \ldots, t_{i-1}, s^{l-1}, t_{i+1}, \ldots, t_{n}\right) \in S^{1}\left(s^{l-1} S\right)^{n+1}=\{0\} .
$$

Since the $t_{j}$ are arbitrary, we conclude that $S^{i-1} S^{l-1} S^{n-i}=\{0\}$, a contradiction. Hence $l=1$ and $S^{i-1} s S^{n-i}=\{0\}$, for every $s \in S$. Thus $S^{n}=\{0\}$.

COROLlaRY 1.5. Let $S$ be a semigroup with zero and no nonzero idempotents. If $S$ is either an $\mathscr{L}_{n}$-semigroup or an $\overline{\mathscr{M}}_{n}^{i, 2}$-semigroup then $S^{n}=\{0\}$.

Definition. Let $S$ be a semigroup. $I=I(S)=\left\{x \mid x \in S, x^{l}=x\right.$ for some positive integer $l \geqq 2\}$. $E=E(S)=\left\{x \mid x \in S, x^{2}=x\right\}$.

LEMMA 1.6. If $S$ is an $\mathscr{L}_{n}$-semigroup or an $\overline{\mathscr{M}}_{n}^{i, 2}$-semigroup then $S E \cup E S \subseteq I$.

Proof. (i) Let $S$ be an $\mathscr{L}_{n}$-semigroup. We will show that $S E \subseteq I$; the proof that $E S \subseteq I$ is similar. Let $a \in S, e \in E$. Then by (1), there exists $f_{2}$ such that $a e=a e \cdots e$ $=f_{2}(a e, e, \ldots, e)=e^{k}(a e)^{l} e^{i}=e^{k}(a e)^{l}$ where $l \geqq 2$ and $k=0$ or $k=1$. If $k=1$, ae= $e(a e)^{l}$ so that $e a e=a e$. Hence $a e=e(a e)^{l}=(e a e)(a e)^{l-1}=(a e)^{l}$. Hence, for either $k=0$ or $k=1$, we obtain $a e=(a e)^{l}$, with $l \geqq 2$. Consequently $a e \in I$.

(ii) Let $S$ be an $\overline{\mathscr{M}}_{n}^{i, 2}$-semigroup. Let $a \in S, e \in E$. From (2) and (3) it follows that $a e=a e \cdots e=e s$ for some $s \in S$. Hence $a e=e a e$. Now letting $a_{j}=e$ for $j \neq i$ and $a_{i}=a e$ we have, again applying (2) and (3), that

$$
e \cdots e(a e) e \cdots e=f_{2}(e, \ldots, a e, \ldots, e)=e^{k}(a e)^{l}
$$

where $k=0$ or 1 and $l \geqq 2$. Hence $a e=e a e=e^{k}(a e)^{l}=\left(e^{k} a e\right)(a e)^{l-1}=(a e)(a e)^{l-1}$ $=\left(a e^{l}\right)$. Thus $a e \in I$ and so $S E \subseteq I$. Similarly $E S \subseteq I$.

Lemma 1.7. Let $S$ be an $\mathscr{L}_{n}$-semigroup or an $\overline{\mathscr{M}}_{n}^{i, 2}$-semigroup. Then $E \subseteq I$ and $I$ is an ideal. In particular, the Rees factor semigroup S/I has no nonzero idempotents.

Proof. Either hypothesis implies that $S$ is periodic and hence $E \neq \varnothing$. Clearly $E \subseteq I$. Let $a \in I, x \in S$. Then there exists $l \geqq 2$ such that $a=a^{l}$. Hence $a^{l-1} \in E$. Consequently $a x=a^{l} x=a^{l-1}(a x) \in I$, by Lemma 1.6 .

\section{Main theorems.}

THEOREM 2.1. Let $S$ be either an $\mathscr{L}_{n}$-semigroup or an $\overline{\mathscr{M}}_{n}^{i, 2}$-semigroup. Then $S^{n}$ is a disjoint union of periodic groups.

Proof. By Lemma $1.7, I$ is an ideal and $S / I$ has no nonzero idempotents. Since $S / I$ is a homomorphic image of $S, S / I$ is either an $\mathscr{L}_{n}$-semigroup or an $\overline{\mathscr{M}}_{n}^{i, 2}$ semigroup. By Corollary $1.5, S^{n} \subseteq I$. Hence for all $x \in S^{n}$, there exists $l \geqq 2$ such 
that $x^{l}=x$. It is well known (cf. [1, p. 23, Exercise 6a]), that this condition implies that $S^{n}$ is a disjoint union of periodic groups.

REMARK. A semigroup $S$ is an $\mathscr{L}_{n}$-semigroup if and only if $S^{n}$ is a disjoint union of periodic groups. Theorem 2.4 shows that a union of periodic groups is not necessarily an $\overline{\mathscr{M}}_{n}^{i, 2}$-semigroup.

Definition. A semigroup $S$ is viable if $a b, b a \in E$ implies $a b=b a$. Idempotents are central in $S$ if $a e=e a$, for every $e \in E, a \in S$.

LEMma 2.2. (i) If $S$ is an $\overline{\mathscr{M}}_{n}^{i, 2}$-semigroup then idempotents are central in $S$.

(ii) For any semigroup $S$, if idempotents are central in $S$, then $S$ is viable.

(iii) Let $S$ be an $\mathscr{L}_{n}$-semigroup. Then $S$ is viable if and only if idempotents are central in $S$ if and only if idempotents commute in $S$.

Proof. (i) Let $e \in E, a \in S$. By (2) and (3), there exists $f_{2}$ such that $e a=e \cdots e a$ $=s e$ for some $s$ in $S$. Hence $e a=e a e$. Similarly $a e=e a e$. Thus $e a=a e$.

(ii) Suppose $a b, b a \in E$. Then $a b=(a b)(a b)=a(b a) b=(b a)(a b)=b(a b) a=(b a)(b a)$ $=b a$.

(iii) By Theorem 2.1, $S^{n}$ is a union of groups. If $S$ is viable then $S^{n}$ is viable. By [3, Theorem 13], idempotents of $S^{n}$ are central in $S^{n}$. Let $e \in E, a \in S$. Then $e=e^{n} ; e a=e^{n-1} a \in S^{n}$. Hence $e a=e(e a)=(e a) e$. Similarly $e a e=a e$. Therefore $e a=a e$, showing idempotents are central in $S$. The converse follows from (ii). If idempotents commute in $S$ then (cf. [1, pp. 126, 127]), idempotents are central in $S^{n}$. The above argument shows idempotents are central in $S$. The following lemma is Lemma 3 of [2].

LEMMA 2.3. Let $S$ be a semigroup with central idempotents. If $a_{1}, a_{2} \in S$ and $e_{1} \in\left\langle a_{1}\right\rangle, e_{2} \in\left\langle a_{2}\right\rangle, e \in\left\langle a_{1} a_{2}\right\rangle$ where $e, e_{1}, e_{2} \in E$, then $e_{1} e_{2}=e$.

THEOREM 2.4. Let $S$ be a semigroup. The following are equivalent.

(i) $S$ is an $\overline{\mathscr{L}}_{n}$-semigroup.

(ii) $S$ is an $\overline{\mathscr{M}}_{n}^{i, 2}$-semigroup.

(iii) $S$ is an $\mathscr{L}_{n}$-semigroup and idempotents commute.

(iv) $S$ is an $\mathscr{L}_{n}$-semigroup with central idempotents.

(v) $S$ is a viable $\mathscr{L}_{n}$-semigroup.

(vi) $S$ is an nth inflation of a semilattice of periodic groups.

Proof. (i) $\Rightarrow$ (ii) a fortiori.

(ii) $\Rightarrow$ (iii). By Theorem 2.1, $S^{n}$ is a disjoint union of periodic groups. Let $a_{1}, \ldots, a_{n} \in S$. Then $a_{1} \cdots a_{n} \in S^{n}$, so there exists $k \geqq 2$ such that $a_{1} \cdots a_{n}=$ $\left(a_{1} \cdots a_{n}\right)^{k}$. Consequently $S$ is an $\mathscr{L}_{n}$-semigroup. By Lemma 2.2(i), idempotents are central in $S$ so surely they commute.

(iii) $\Rightarrow$ (iv) follows by Lemma 2.2(iii).

(iv) $\Rightarrow$ (v). By Lemma 2.2(ii). $S$ is viable.

(v) $\Rightarrow$ (vi). By Theorem 2.1, $S^{n}$ is a disjoint union of periodic groups. By Lemma 2.2(iii), idempotents are central in $S$, so certainly idempotents are central in $S^{n}$. 
Hence (cf. [1, Theorem 4.11]), $S^{n}$ is a semilattice of periodic groups. We show that $S$ is an $n$th inflation of $S^{n}$. Define $\theta: S \rightarrow S^{n}$ by: $a \theta=a e$ where $e \in\langle a\rangle$. Clearly $\theta$ is the identity on $S^{n}$. Also if $a, b \in S, e_{1} \in\langle a\rangle, e_{2} \in\langle b\rangle$, then by Lemma 2.3, $e_{1} e_{2} \in\langle a b\rangle$. Hence $(a b) \theta=a b e_{1} e_{2}=\left(a e_{1}\right)\left(b e_{2}\right)=(a \theta)(b \theta)$. Hence $\theta$ is a homomorphism. If $a_{1}, \ldots, a_{n} \in S$, then $\left(a_{1} \theta\right) \cdots\left(a_{n} \theta\right)=\left(a_{1} \cdots a_{n}\right) \theta=a_{1} \cdots a_{n}$ since $\theta$ is the identity on $S^{n}$.

(vi) $\Rightarrow$ (i). Let $G$ be a periodic group with identity $e$ and let $a_{1}, \ldots, a_{n} \in G$. There exist integers $i>1, j>1, k>1$ such that $a_{1}^{i}=e, a_{n}^{j}=e,\left(a_{1} \cdots a_{n}\right)^{k}=\left(a_{1} \cdots a_{n}\right)$. Thus $a_{1} \cdots a_{n}=a_{n}^{j}\left(a_{1} \cdots a_{n}\right)^{k} a_{1}^{i}$. Consequently $G$ is an $\overline{\mathscr{L}}_{n}$-semigroup. Thus by Corollary $1.2, S$ is an $\overline{\mathscr{L}}_{n}$-semigroup.

Corollary 2.5. Let $\mathscr{X} \subseteq \overline{\mathscr{M}}_{n}^{i, 2}$. Then $S$ is an $\mathscr{X}$-semigroup if and only if $S$ is an nth inflation of a semilatice of $\mathscr{X}$-groups.

Proof. If $S$ is an $\mathscr{X}$-semigroup, then it is an $\overline{\mathscr{M}}_{n}^{i, 2}$-semigroup. Hence $S$ is an $n$th inflation of a semilattice of groups. Each of these groups, being a subgroup of $S$, is an $\mathscr{X}$-group. The converse follows from Corollary 1.2 .

CoRollary 2.6. Let $\mathscr{X} \subseteq \mathscr{M}_{n}^{i, 2}$. Then $S^{n-1}$ is in the center of $S$ for every $\mathscr{X}$ semigroup $S$ if and only if

(i) $\mathscr{X} \subseteq \overline{\mathscr{M}}_{n}^{i, 2}$, and

(ii) every $\mathscr{X}$-group is abelian.

Proof. The necessity of (ii) is clear. If (i) does not hold then there exists $f=$ $x_{1} \cdots x_{n}-f_{2} \in \mathscr{X}$ such that $f_{2}$ starts with $x_{1}$ or ends with $x_{n}$. Let $S_{1}$ (respectively $S_{2}$ ) be a nontrivial right-(left-)zero semigroup. Then either $S_{1}$ or $S_{2}$ is an $\{f\}$-semigroup and hence an $\mathscr{X}$-semigroup. But $S_{1}^{n-1}=S_{1}\left(S_{2}^{n-1}=S_{2}\right)$ which is not in the center of $S_{1}\left(S_{2}\right)$.

Conversely assume (i) and (ii) hold. By Corollary 2.5, $S$ is an $n$th inflation of a semilattice of $\mathscr{X}$-groups $G_{\alpha}$. By (ii) each $G_{\alpha}$ is abelian and hence satisfies every permutation identity in $n$-variables. By Corollary 1.2 , letting $\mathscr{X}$ be any single permutation identity in $n$-variables, $S$ itself satisfies every permutation identity in $n$-variables. In particular $S^{n-1}$ is in the center.

REMARK. In Corollary 2.6, we can replace the words " $S^{n-1}$ is in the center of $S$ " with " $S$ satisfies every permutation identity in $n$-variables."

Theorem 2.4 and Corollary 2.6 yield the main theorem and corollary of Tamura [4], when $n=2$ and $\mathscr{X}=\left\{x_{1} x_{2}-f\left(x_{1}, x_{2}\right)\right\}, f$ a fixed monomial in $x_{1}$ and $x_{2}$. In addition Theorem 2.4 generalizes the main theorem of [2].

\section{Examples and problems.}

ExAmple 1. Theorem 2.1 is not true for $\mathscr{M}_{n}^{i, \infty}$-semigroups. Let $S$ be the multiplicative semigroup of $2 \times 2$ matrices over $G F(2)$ consisting of $\left\{\left[\begin{array}{ll}0 & 0 \\ 0 & 0\end{array}\right],\left[\begin{array}{ll}1 & 0 \\ 1 & 0\end{array}\right],\left[\begin{array}{ll}0 & 1 \\ 0 & 1\end{array}\right],\left[\begin{array}{ll}1 & 1 \\ 1 & 1\end{array}\right]\right\}$. Then $S$ satisfies $x_{1} x_{2}=x_{1}^{\alpha} x_{2}$ for all $\alpha \geqq 2$ and hence $S$ is an $\mathscr{M}_{2}^{1, \infty}$-semigroup. However $S^{2}=S$ is not a union of groups. 
ExAMPLE 2. Theorem 1.1 is not true if the condition that every $T_{\alpha}$ has an identity is deleted. Let $n=2, \mathscr{X}=\left\{x_{1} x_{2}-x_{2} x_{1}\right\}$. Let $S$ be the semigroup given by:

\begin{tabular}{c|c|c|c} 
& 0 & $a$ & $b$ \\
\hline 0 & 0 & 0 & 0 \\
$a$ & 0 & 0 & 0 \\
$b$ & 0 & $a$ & $b$
\end{tabular}

Let $T_{1}=\{0, a\}, T_{2}=\{b\}$. Then $T_{1}$ and $T_{2}$ are $\mathscr{X}$-semigroups and $S$ is a semilattice of $T_{1}$ and $T_{2}$. But $S$ is not an $\mathscr{X}$-semigroup.

PRoBlem 1. Theorems 2.1 and 2.4 characterize $\mathscr{L}_{n}$-semigroups and $\overline{\mathscr{M}}_{n}^{i, 2}$-semigroups. Characterize other $\mathscr{X}$-semigroups. Study semigroups which are $\{f\}-$ semigroups for some $f \in \mathscr{V}_{n}$.

Problem 2. Is there a "nice" subset $\mathscr{X}$ of $\mathscr{V}_{n}$ such that $S$ is an $\mathscr{X}$-semigroup if and only if $S^{n}$ is a band of groups?

\section{REFERENCES}

1. A. H. Clifford and G. B. Preston, The algebraic theory of semigroups. Vol. 1, Math. Surveys, no. 7, Amer. Math. Soc., Providence, R. I., 1961. MR 24 \#A2627.

2. M. S. Putcha and J. Weissglass, Semigroups satisfying variable identities, Semigroup Forum 3 (1971), 64-67.

3. - A semilattice decomposition into semigroups having at most one idempotent, Pacific J. Math. 38 (1971).

4. T. Tamura, Semigroups satisfying identity $x y=f(x, y)$, Pacific J. Math. 31 (1969), 513-521. MR 41 \#5528.

5. E. J. Tully, Semigroups satisfying an identity of the form $x y=y^{m} x^{n}$ (to appear).

Department of Mathematics, University of California, Santa Barbara, California 93106

Current address (Mohan S. Putcha): University of California, Berkeley, California 94720 